\title{
Recovery of Finite Missing Samples in Two-channel Oversampling
}

\author{
J. M. Kim \\ Division of Applied Math., KAIST, 373-1 Guseongdong \\ Yuseonggu, Daejeon, Korea \\ franzkim@amath.kaist.ac.kr \\ K. H. Kwon \\ Division of Applied Math., KAIST, 373-1 Guseongdong \\ Yuseonggu, Daejeon, Korea \\ khkwon@amath.kaist.ac.kr
}

\begin{abstract}
It is well known that any finite missing samples of a band-limited signal can be recovered when the signal is oversampled at a rate higher than the minimum Nyquist rate. This paper handles the problem of recovering any finite missing samples when a band-limited signal is oversampled through two channels. We find sufficient conditions under which any finitely many missing samples can be recovered when samples are missing either from a single channel or both channels.
\end{abstract}

Key words and phrases : Oversampling, two-channel sampling, recovery of missing samples, sampling theory

2000 AMS Mathematics Subject Classification - 94A20

\section{Introduction}

When a band-limited signal is oversampled at a rate higher than the minimum Nyquist rate, the samples thus obtained are not independent but has some redundancy. Hence, through oversampling any finite missing samples can be recovered from the remaining known samples $([1,2,7,9])$. (See also [5] for an abstract setting of the oversampling and recovering of missing samples in general reproducing kernel Hilbert spaces.) Recently, Santos and Ferreira [9] considered the problem of recovering missing samples in two-channel oversampling involving a signal and its derivative. In [9] it is shown that any finite missing samples can always be recovered if these missing samples occur either in the signal itself or its derivative. In this work, we consider arbitrary two-channeling of a 
band-limited signal and find sufficient conditions under which we can recover any finite missing samples when they occur in a single channel or both channels. We also give several examples illustrating our results.

\section{Two-channel oversampling}

Let $P W_{\pi}$ be the Paley-Wiener space of band-limited signals, consisting of square integrable (i.e., finite energy) signals $f(t)$, of which its Fourier transform

$$
\hat{f}(\xi):=\mathcal{F}(f)(\xi)=\frac{1}{\sqrt{2 \pi}} \int_{-\infty}^{\infty} f(t) e^{-i t \xi} d t
$$

has a bounded support in $[-\pi, \pi]$.

Let $a_{j}(\xi)$ for $j=1,2$ be bounded measurable functions on $[-\pi, \pi]$ (i.e., prefilters) satisfying

$$
|\operatorname{det} a(\xi)| \geq \alpha \text { a.e. on }[-\pi, 0]
$$

for some constant $\alpha>0$, where

$$
a(\xi):=\left[\begin{array}{ll}
a_{1}(\xi) & a_{1}(\xi+\pi) \\
a_{2}(\xi) & a_{2}(\xi+\pi)
\end{array}\right]
$$

is the so-called transfer matrix. Let

$$
c_{j}(f)(t):=\mathcal{F}^{-1}\left[a_{j}(\xi) \hat{f}(\xi)\right]=\frac{1}{\sqrt{2 \pi}} \int_{-\pi}^{\pi} a_{j}(\xi) \hat{f}(\xi) e^{i t \xi} d \xi
$$

be the channeled signals of $f(t)$. Then, we have a two-channel sampling series expansion.

Theorem 2.1. (cf. [3,4,8]) Any signal $f(t)$ in $P W_{\pi}$ can be expanded as a sampling series

$$
f(t)=\sum_{j=1}^{2} \sum_{n} c_{j}(f)(2 n) S_{j, n}(t)
$$

where $\left\{S_{j, n}(t): j=1,2\right.$ and $\left.n \in \mathbb{Z}\right\}$ is a Riesz basis of $P W_{\pi}$. The series (2.3) converges not only in $P W_{\pi}$ but also absolutely and uniformly on $\mathbb{R}$.

Taking the Fourier transform of (2.3), we obtain

$$
\hat{f}(\xi)=\sum_{j=1}^{2} \sum_{n}\left\langle\hat{f}(\xi), \frac{1}{\sqrt{2 \pi}} \overline{a_{j}(\xi)} e^{-i 2 n \xi}\right\rangle_{L^{2}[-\pi, \pi]} \phi_{j, n}(\xi)
$$

where $\left\{\phi_{j, n}(\xi)=\hat{S}_{j, n}(\xi): j=1,2\right.$ and $\left.n \in \mathbb{Z}\right\}$ is a Riesz basis of $L^{2}[-\pi, \pi]$, of which the dual is

$$
\left\{\phi_{j, n}^{*}(\xi):=\frac{1}{\sqrt{2 \pi}} \overline{a_{j}(\xi)} e^{-i 2 n \xi}: j=1,2 \text { and } n \in \mathbb{Z}\right\} .
$$


Then we have (cf. [6])

$$
\phi_{j, n}(\xi)=\sqrt{\frac{2}{\pi}} D^{-1}\left(b_{j}(\xi)\right) e^{-i 2 n \xi}
$$

where $b_{j}(\xi)$ is the $j$-th column of

$$
b(\xi):=a(\xi)^{-1}=\left[\begin{array}{ll}
b_{1,1}(\xi) & b_{1,2}(\xi) \\
b_{2,1}(\xi) & b_{2,2}(\xi)
\end{array}\right]
$$

and $D: L^{2}[-\pi, \pi] \longrightarrow L^{2}[-\pi, 0]^{2}$ is the unitary operator defined by

$$
D(\phi)(\xi)=\left[\begin{array}{c}
\phi_{1}(\xi) \\
\phi_{2}(\xi)
\end{array}\right]=\left[\begin{array}{c}
\phi(\xi) \\
\phi(\xi+\pi)
\end{array}\right] .
$$

For later use we note that as a Riesz basis of $L^{2}[-\pi, \pi],\left\{\phi_{j, n}(\xi)\right\}$ must be an isomorphic image of an orthonormal basis of $L^{2}[-\pi, \pi]$. To be precise, we have

$$
\phi_{j, n}(\xi)=\left(T^{*}\right)^{-1}\left(e_{j, n}(\xi)\right)
$$

where $\left\{e_{j, n}(\xi)\right\}$ is the orthonormal basis of $L^{2}[-\pi, \pi]$ given by

$$
e_{1, n}(\xi)=\frac{1}{\sqrt{\pi}} e^{-i 2 n \xi} \chi_{[-\pi, 0]}(\xi), e_{2, n}(\xi)=\frac{1}{\sqrt{\pi}} e^{-i 2 n \xi} \chi_{[0, \pi]}(\xi)
$$

and

$$
T=\frac{1}{\sqrt{2 \pi}} D^{-1} \circ a(\xi)^{*} \circ D: L^{2}[-\pi, \pi] \longrightarrow L^{2}[-\pi, \pi]
$$

is an isomorphism where

$$
\chi_{A}(\xi)= \begin{cases}1 & \text { if } \xi \in A \\ 0 & \text { if } \xi \notin A\end{cases}
$$

is the characteristic function of set $A$.

For a measurable set $E$ in $\mathbb{R}$, let $|E|$ be the Lebesgue measure of $E$. We now let $B$ be a bounded band region, which consists of finitely many disjoint closed intervals and we consider signals in

$$
P W_{B}:=\left\{f(t) \in L^{2}(\mathbb{R}): \operatorname{supp} \hat{f}(\xi) \subseteq B\right\} .
$$

We may and shall assume that $B \subsetneq[-\pi, \pi]$ so that the total length $|B|$ of $B$ is less than $2 \pi$.

Viewing a signal $f(t)$ in $P W_{B}$ as a signal in $P W_{\pi}$, we can expand $f(t)$ as in (2.3). Then we also have

$$
\hat{f}(\xi)=\sum_{k=1}^{2} \sum_{n} c_{k}(f)(2 n) \phi_{k, n}(\xi)=\sum_{k=1}^{2} \sum_{n} c_{k}(f)(2 n) \phi_{k, n}(\xi) h(\xi)
$$


for any bounded measurable function $h(\xi)$ on $[-\pi, \pi]$ with $h(\xi)=1$ on $B$. By taking the inverse Fourier transform on (2.6), we obtain an oversampling series

$$
f(t)=\sum_{k=1}^{2} \sum_{n} c_{k}(f)(2 n) T_{k, n}(t), t \in \mathbb{R},
$$

where $T_{k, n}(t):=\frac{1}{\sqrt{2 \pi}} \int_{-\pi}^{\pi} \phi_{k, n}(\xi) h(\xi) e^{i t \xi} d \xi$. Note that $\left\{T_{k, n}(t): k=1,2\right.$ and $n \in$ $\mathbb{Z}\}$ may not be a Riesz basis of $P W_{B}$ in general.

Our main problem is as follows. Assume that finitely many samples

$$
\left\{c_{1}(f)(2 m): m \in X_{1}\right\} \text { and }\left\{c_{2}(f)(2 n): n \in X_{2}\right\}
$$

are missing, where $X_{1}$ and $X_{2}$ are any finite index sets. Under what conditions can we recover these missing samples?

By (2.2) and (2.6), we have

$$
c_{j}(f)(t)=\sum_{k=1}^{2} \sum_{n} c_{k}(f)(2 n)\left\langle\phi_{k, n}(\xi) h(\xi), \frac{1}{\sqrt{2 \pi}} \overline{a_{j}(\xi)} e^{-i t \xi}\right\rangle_{L^{2}[-\pi, \pi]}
$$

so that

$$
\begin{aligned}
c_{j}(f)(2 m) & =\sum_{k=1}^{2} \sum_{n} c_{k}(f)(2 n)\left\langle\phi_{k, n}(\xi) h(\xi), \phi_{j, m}^{*}(\xi)\right\rangle_{L^{2}[-\pi, \pi]} \\
& =\sum_{k=1}^{2} \sum_{n} c_{k}(f)(2 n) R_{j, k}(m, n)
\end{aligned}
$$

where $R_{j, k}(m, n)=\left\langle\phi_{k, n}(\xi) h(\xi), \phi_{j, m}^{*}(\xi)\right\rangle_{L^{2}[-\pi, \pi]}$. When $X_{1} \neq \phi$ or $X_{2} \neq \phi$, we let

$$
X_{1}=\left\{m_{1}, m_{2}, \cdots, m_{M}\right\} \text { or } X_{2}=\left\{n_{1}, n_{2}, \cdots, n_{N}\right\} .
$$

Then

$$
\begin{aligned}
c_{1}(f)\left(2 m_{j}\right)= & \sum_{k=1}^{M} c_{1}(f)\left(2 m_{k}\right) R_{1,1}\left(m_{j}, m_{k}\right) \\
& +\sum_{k=1}^{N} c_{2}(f)\left(2 n_{k}\right) R_{1,2}\left(m_{j}, n_{k}\right)+g_{1, j}, 1 \leq j \leq M \\
c_{2}(f)\left(2 n_{j}\right)= & \sum_{k=1}^{M} c_{1}(f)\left(2 m_{k}\right) R_{2,1}\left(n_{j}, m_{k}\right) \\
& +\sum_{k=1}^{N} c_{2}(f)\left(2 n_{k}\right) R_{2,2}\left(n_{j}, n_{k}\right)+g_{2, j}, 1 \leq j \leq N
\end{aligned}
$$


where $\left\{g_{1, j}\right\}_{j=1}^{M}$ and $\left\{g_{2, j}\right\}_{j=1}^{N}$ are known terms. Set

$$
\mathbf{f}=\left[c_{1}(f)\left(2 m_{1}\right), \cdots, c_{1}(f)\left(2 m_{M}\right), c_{2}(f)\left(2 n_{1}\right), \cdots, c_{2}(f)\left(2 n_{N}\right)\right]^{T}
$$

and

$$
\mathbf{g}=\left[g_{1,1}, \cdots, g_{1, M}, g_{2,1}, \cdots, g_{2, N}\right]^{T} .
$$

Then following the same procedure as in $[1,9]$ we can rewrite the above as a matrix form:

$$
(I-S) \mathbf{f}=\mathbf{g}
$$

where $S=\left[\begin{array}{ll}S_{1,1} & S_{1,2} \\ S_{2,1} & S_{2,2}\end{array}\right]$ is a block matrix with

$$
\begin{aligned}
& S_{1,1}=\left[R_{1,1}\left(m_{j}, m_{k}\right)\right]_{j, k=1}^{M}, S_{1,2}=\left[R_{1,2}\left(m_{j}, n_{k}\right)\right]_{j=1, k=1}^{M N}, \\
& S_{2,1}=\left[R_{2,1}\left(n_{j}, m_{k}\right)\right]_{j=1, k=1}^{N M}, S_{2,2}=\left[R_{2,2}\left(n_{j}, n_{k}\right)\right]_{j, k=1}^{N} .
\end{aligned}
$$

For later use, we note that

$$
\begin{aligned}
R_{j, k}(m, n) & =\frac{1}{\pi} \int_{-\pi}^{\pi} a_{j}(\xi) D^{-1}\left(b_{k}\right)(\xi) h(\xi) e^{2 i(m-n) \xi} d \xi \\
& =\frac{1}{\pi} \int_{-\pi}^{0} G_{j, k}(\xi) e^{2 i(m-n) \xi} d \xi
\end{aligned}
$$

where $\Delta=\operatorname{det} a(\xi)$ and (cf. (2.1))

$$
\begin{aligned}
G_{1,1}(\xi) & =a_{1}(\xi) b_{1,1}(\xi)[h(\xi)-h(\xi+\pi)]+h(\xi+\pi) \\
& =\frac{1}{\Delta} a_{1}(\xi) a_{2}(\xi+\pi)[h(\xi)-h(\xi+\pi)]+h(\xi+\pi) \\
G_{1,2}(\xi) & =a_{1}(\xi) b_{1,2}(\xi)[h(\xi)-h(\xi+\pi)] \\
& =\frac{-1}{\Delta} a_{1}(\xi) a_{1}(\xi+\pi)[h(\xi)-h(\xi+\pi)] \\
G_{2,1}(\xi) & =a_{2}(\xi) b_{1,1}(\xi)[h(\xi)-h(\xi+\pi)] \\
& =\frac{1}{\Delta} a_{2}(\xi) a_{2}(\xi+\pi)[h(\xi)-h(\xi+\pi)] \\
G_{2,2}(\xi) & =a_{2}(\xi) b_{1,2}(\xi)[h(\xi)-h(\xi+\pi)]+h(\xi+\pi) \\
& =\frac{-1}{\Delta} a_{1}(\xi+\pi) a_{2}(\xi)[h(\xi)-h(\xi+\pi)]+h(\xi+\pi) .
\end{aligned}
$$

Note that the missing samples $\mathbf{f}$ can be uniquely recovered from (2.7) if $I-S$ is invertible. In particular, if $\langle S w, w\rangle \neq\|w\|^{2}$ for any $w=\left(u_{1}, \cdots, u_{M}, v_{1}, \cdots, v_{N}\right)^{T}$ in $\mathbb{C}^{M+N} \backslash\{0\}$, then 1 is not an eigenvalue of the matrix $S$ and, consequently, the matrix $I-S$ is invertible. On the other hand, $\langle S w, w\rangle=$

$$
\int_{-\pi}^{0}\left[G_{1,1}(\xi)|u(\xi)|^{2}+G_{1,2}(\xi) u(\xi) \overline{v(\xi)}+G_{2,1}(\xi) \overline{u(\xi)} v(\xi)+G_{2,2}(\xi)|v(\xi)|^{2}\right] d \xi
$$


where $u(\xi)=\sum_{k=1}^{M} \overline{u_{k}}\left(\frac{1}{\sqrt{\pi}} e^{2 i m_{k} \xi}\right), v(\xi)=\sum_{k=1}^{N} \overline{v_{k}}\left(\frac{1}{\sqrt{\pi}} e^{2 i n_{k} \xi}\right)$. Then

$$
\int_{-\pi}^{0}|u(\xi)|^{2} d \xi=\|u\|^{2}=\sum_{k=1}^{M}\left|u_{k}\right|^{2}, \quad \int_{-\pi}^{0}|v(\xi)|^{2} d \xi=\|v\|^{2}=\sum_{k=1}^{M}\left|v_{k}\right|^{2}
$$

since $\left\{\frac{1}{\sqrt{\pi}} e^{2 i n \xi}\right\}$ is an orthonormal basis of $L^{2}[-\pi, 0]$.

\section{Missing samples from a single channel}

In Section 3 we take $B=[-r \pi, r \pi]$ except in Example 3.3 and assume that either $X_{1} \neq \phi$ and $X_{2}=\phi$ or $X_{1}=\phi$ and $X_{2} \neq \phi$. As it is pointed out in [9], when $0<r<\frac{1}{2}, P W_{B} \subset P W_{\frac{\pi}{2}}$ so that any missing samples $\left\{c_{1}(f)(2 m): m \in X_{1}\right\}$ or $\left\{c_{2}(f)(2 n): n \in X_{2}\right\}$ can be recovered from the oversampling of $c_{1}(f)(t)$ or $c_{2}(f)(t)$ in $P W_{\frac{\pi}{2}}$ separately. Hence, we also assume $\frac{1}{2} \leq r<1$ and $X_{1} \neq \phi$ and $X_{2}=\phi$. Then the missing samples $\mathbf{f}_{1}=\left[c_{1}(f)\left(2 m_{1}\right), \cdots, c_{1}(f)\left(2 m_{M}\right)\right]^{T}$ satisfies

$$
\left(I-S_{1,1}\right) \mathbf{f}_{1}=\mathbf{g}_{1}
$$

where $\mathbf{g}_{1}=\left(g_{1,1}, \cdots, g_{1, M}\right)^{T}$ is known and

$$
S_{1,1}=\frac{1}{\pi}\left[\int_{-\pi}^{0} G_{1,1}(\xi) e^{2 i\left(m_{j}-m_{k}\right) \xi} d \xi\right]_{j, k=1}^{M} .
$$

Since $h(\xi)=1$ on $B$, we have from $(2.8)$

$$
G_{1,1}(\xi)= \begin{cases}1+a_{1}(\xi) b_{1,1}(\xi)[h(\xi)-1] & ,-\pi \leq \xi<-r \pi \\ 1 & ,-r \pi \leq \xi<-(1-r) \pi \\ h(\xi+\pi)\left(1-a_{1}(\xi) b_{1,1}(\xi)\right)+a_{1}(\xi) b_{1,1}(\xi) & ,-(1-r) \pi \leq \xi \leq 0 .\end{cases}
$$

Let

$$
\begin{aligned}
& E_{1}=\left\{-\pi \leq \xi<-r \pi: a_{1}(\xi) b_{1,1}(\xi) \neq 0\right\} \\
& E_{2}=\left\{-(1-r) \pi \leq \xi \leq 0: a_{1}(\xi) b_{1,1}(\xi) \neq 1\right\}
\end{aligned}
$$

and $E=E_{1} \cup E_{2}$.

Theorem 3.1. Let $B=[-r \pi, r \pi]$ with $\frac{1}{2} \leq r<1$. If $|E|>0$, then any finite missing samples $\mathbf{f}_{1}$ can be recovered.

Proof. First, note that $G_{1,1}(\xi)=1$ on $[-\pi, 0] \backslash E$ and

$$
\begin{aligned}
& E_{1}=\left\{-\pi \leq \xi \leq-r \pi: a_{1}(\xi) a_{2}(\xi+\pi) \neq 0\right\} \\
& E_{2}=\left\{-(1-r) \pi \leq \xi \leq 0: a_{1}(\xi+\pi) a_{2}(\xi) \neq 0\right\} .
\end{aligned}
$$


Since $|E|>0$, there is an integer $k \geq 1$ such that $|F|>0$, where $F=F_{1} \cup F_{2}$ and

$$
\begin{aligned}
& F_{1}=\left\{-\pi \leq \xi<-r \pi:\left|a_{1}(\xi) a_{2}(\xi+\pi)\right| \geq \frac{1}{k}\right\} \\
& F_{2}=\left\{-(1-r) \pi \leq \xi \leq 0:\left|a_{1}(\xi+\pi) a_{2}(\xi)\right| \geq \frac{1}{k}\right\}
\end{aligned}
$$

We now define $h(\xi)$ on $[-\pi, \pi]$ as

$$
h(\xi)= \begin{cases}\frac{a_{1}(\xi+\pi) a_{2}(\xi)}{a_{1}(\xi) a_{2}(\xi+\pi)} & \text { on } F_{1} \text { if }\left|F_{1}\right|>0 \\ \frac{a_{1}(\xi-\pi) a_{2}(\xi)}{a_{1}(\xi) a_{2}(\xi-\pi)} & \text { on } F_{2}+\pi \text { if }\left|F_{2}\right|>0 \\ 1 & \text { on }[-\pi, \pi] \backslash\left(F_{1} \cup\left(F_{2}+\pi\right)\right) .\end{cases}
$$

Then $h(\xi)$ is a bounded measurable function on $[-\pi, \pi]$ with $h(\xi)=1$ on $B$ for which we have (cf. (3.2))

$$
G_{1,1}(\xi)= \begin{cases}0 & \text { on } F \\ 1 & \text { on }[-\pi, 0] \backslash F .\end{cases}
$$

Hence, for any $u=\left(u_{1}, \cdots, u_{M}\right)^{T} \in \mathbb{C}^{M} \backslash\{0\}$,

$$
\left\langle S_{1,1} u, u\right\rangle=\int_{-\pi}^{0} G_{1,1}(\xi)|u(\xi)|^{2} d \xi=\int_{F^{c}}|u(\xi)|^{2} d \xi<\int_{-\pi}^{0}|u(\xi)|^{2} d \xi=\|u\|^{2}
$$

where $u(\xi):=\sum_{k=1}^{M} \overline{u_{k}}\left(\frac{1}{\sqrt{\pi}} e^{2 i m_{k} \xi}\right)$.

Hence, 1 is not an eigenvalue of $S_{1,1}$, that is, $I-S_{1,1}$ is nonsingular so that the equation (3.1) is uniquely solvable for missing samples $\mathbf{f}_{1}$.

When $X_{1}=\phi$ and $X_{2} \neq \phi$, the missing samples $\mathbf{f}_{2}=\left[c_{2}(f)\left(2 n_{1}\right), \cdots, c_{2}(f)\left(2 n_{N}\right)\right]^{T}$ satisfies

$$
\left(I-S_{2,2}\right) \mathbf{f}_{2}=\mathbf{g}_{2}
$$

where

$$
S_{2,2}=\frac{1}{\pi}\left[\int_{-\pi}^{0} G_{2,2}(\xi) e^{2 i\left(n_{j}-n_{k}\right) \xi} d \xi\right]_{j, k=1}^{N}
$$

and

$G_{2,2}(\xi)= \begin{cases}1+a_{2}(\xi) b_{1,2}(\xi)[h(\xi)-1] & ,-\pi \leq \xi<-r \pi \\ 1 & ,-r \pi \leq \xi<-(1-r) \pi \\ h(\xi+\pi)\left(1-a_{2}(\xi) b_{1,2}(\xi)\right)+a_{2}(\xi) b_{1,2}(\xi) & ,-(1-r) \pi \leq \xi \leq 0 .\end{cases}$

Hence, by the same reasoning as above, we have: 
Theorem 3.2. Let $B=[-r \pi, r \pi]$ with $\frac{1}{2} \leq r<1$. If $|E|>0$, where

$$
\begin{aligned}
E= & \left\{-\pi \leq \xi<-r \pi: a_{1}(\xi+\pi) a_{2}(\xi) \neq 0\right\} \\
& \cup\left\{-(1-r) \pi \leq \xi \leq 0: a_{1}(\xi) a_{2}(\xi+\pi) \neq 0\right\},
\end{aligned}
$$

then any finitely many missing samples $\mathbf{f}_{2}$ can be recovered.

In the case $X_{1} \neq \phi$ and $X_{2}=\phi$ (resp. $X_{1}=\phi$ and $X_{2} \neq \phi$ ), we may take $h(\xi)=\chi_{B}(\xi)$ to recover missing samples $\mathbf{f}_{1}$ (resp. $\mathbf{f}_{2}$ ) provided that

$$
|E|>0 \text { and } 0 \leq a_{1}(\xi) b_{1,1}(\xi) \leq 1\left(\text { resp. } 0 \leq a_{2}(\xi) b_{1,2}(\xi) \leq 1\right) \text { on } E,
$$

where $E$ is the same as in Theorem 3.1 (resp. Theorem 3.2).

Example 3.1. (derivative sampling [9]) Take $a_{1}(\xi)=1, a_{2}(\xi)=i \xi$ on $[-\pi, \pi]$ so that $c_{1}(f)(t)=f(t), c_{2}(f)(t)=f^{\prime}(t)$. Then we have with $h(\xi)=\chi_{B}(\xi)$

$$
G_{1,1}(\xi)= \begin{cases}\frac{-\xi}{\pi} & ,-\pi \leq \xi<-r \pi \\ 1 & ,-r \pi \leq \xi<-(1-r) \pi \\ 1+\frac{\xi}{\pi} & ,-(1-r) \pi \leq \xi \leq 0\end{cases}
$$

and

$$
G_{2,2}(\xi)= \begin{cases}1+\frac{\xi}{\pi} & ,-\pi \leq \xi<-r \pi \\ 1 & ,-r \pi \leq \xi<-(1-r) \pi \\ \frac{-\xi}{\pi} & ,-(1-r) \pi \leq \xi \leq 0 .\end{cases}
$$

Hence, the condition (3.3) holds for both $\mathbf{f}_{1}$ and $\mathbf{f}_{2}$ so that $\mathbf{f}_{1}$ or $\mathbf{f}_{2}$ can be recovered.

Example 3.2. (Hilbert transform sampling) Take $a_{1}(\xi)=1, a_{2}(\xi)=-i \operatorname{sgn} \xi$ on $[-\pi, \pi]$ so that $c_{1}(f)(t)=f(t), c_{2}(f)(t)=\tilde{f}(t)$ is the Hilbert transform of $f(t)$. Then we have with $h(\xi)=\chi_{B}(\xi)$

$$
G_{1,1}(\xi)=G_{2,2}(\xi)= \begin{cases}\frac{1}{2} \quad, \quad-\pi \leq \xi<-r \pi \\ 1 \quad,-r \pi \leq \xi<-(1-r) \pi \\ \frac{1}{2} \quad,-(1-r) \pi \leq \xi \leq 0 .\end{cases}
$$

Hence, the condition (3.3) holds for both $\mathbf{f}_{1}$ and $\mathbf{f}_{2}$ so that $\mathbf{f}_{1}$ or $\mathbf{f}_{2}$ can be recovered.

Example 3.3. (negative example) Take

$$
B=\left[-r \pi,-\frac{1}{2} \pi\right] \cup[-(1-r) \pi,(1-r) \pi] \cup\left[\frac{1}{2} \pi, r \pi\right]
$$


with $\frac{1}{2}<r<1$ and $a_{1}(\xi)=\chi_{B}(\xi), a_{2}(\xi)=\chi_{B^{c}}(\xi)$. Then

$$
a(\xi)= \begin{cases}{\left[\begin{array}{ll}
1 & 0 \\
0 & 1
\end{array}\right]} & \text { on }[-\pi, 0] \cap B \\
{\left[\begin{array}{ll}
0 & 1 \\
1 & 0
\end{array}\right]} & \text { on }[-\pi, 0] \cap B^{c}\end{cases}
$$

so that $|\operatorname{det} a(\xi)|=1>0$ on $[-\pi, 0]$. Hence, $b(\xi)=a(\xi)$ so that $G_{1,1}(\xi)=1$ on $[-\pi, 0]$ for any bounded measurable function $h(\xi)$ on $[-\pi, \pi]$ with $h(\xi)=1$ on $B$. Then any finite missing samples $\mathbf{f}_{1}$ cannot be recovered for any choice of $h(\xi)$ since $S_{1,1}=I$. On the other hand, if $h(\xi)=\chi_{B}(\xi)$, then $G_{2,2}(\xi)=0$ on $[-\pi, 0]$ so that any finite missing samples $\mathbf{f}_{2}$ can be recovered.

Now we construct an example for which missing samples can be recovered only by a suitable choice of $h(\xi)$ other than $\chi_{B}(\xi)$.

Example 3.4. Take $B=[-r \pi, r \pi]$ and $a_{j}(\xi)(i=1,2)$ on $[-\pi, \pi]$ as

$$
\begin{aligned}
& a_{1}(\xi)= \begin{cases}2 & \text { on }[-s \pi,(1-s) \pi) \\
1 & \text { otherwise }\end{cases} \\
& a_{2}(\xi)= \begin{cases}1 & \text { on }[-s \pi,(1-s) \pi) \\
2 & \text { otherwise, }\end{cases}
\end{aligned}
$$

where $\frac{1}{2}<r<s<1$. Then

$$
a(\xi)= \begin{cases}{\left[\begin{array}{ll}
1 & 2 \\
2 & 1
\end{array}\right]} & \text { on }[-\pi,-s \pi) \\
{\left[\begin{array}{ll}
2 & 1 \\
1 & 2
\end{array}\right]} & \text { on }[-s \pi, 0]\end{cases}
$$

and

$$
b(\xi)= \begin{cases}\frac{1}{3}\left[\begin{array}{rr}
-1 & 2 \\
2 & -1
\end{array}\right] & \text { on }[\pi,-s \pi) \\
\frac{1}{3}\left[\begin{array}{rr}
2 & -1 \\
-1 & 2
\end{array}\right] & \text { on }[-s \pi, 0] .\end{cases}
$$

Note that $|\operatorname{det} A(\xi)|=3$ on $[-\pi, 0]$. We now assume that a single sample $c_{1}(f)(2 m)(m \in \mathbb{Z})$ is missing. The equation $(3.1)$ becomes $\left(1-S_{1,1}\right) c_{1}(f)(2 m)=$ $g$, where $g$ is known and $S_{1,1}=\frac{1}{\pi} \int_{-\pi}^{0} G_{1,1}(\xi) d \xi$. Hence, the missing sample $c_{1}(f)(2 m)$ can be recovered if and only if $S_{1,1} \neq 1$. In this example the set $E$ in Theorem 3.1 is $E=[-\pi,-r \pi) \cup[-(1-r) \pi, 0]$ so that by Theorem 3.1 the 
missing sample $c_{1}(f)(2 m)$ can be always recovered by a suitable choice of $h(\xi)$. In fact, for any bounded measurable function $h(\xi)$ on $[-\pi, \pi]$ with $h(\xi)=1$ on $B$, we have from (3.2)

$$
\begin{aligned}
\int_{-\pi}^{0} G_{1,1}(\xi) d \xi= & \left(\int_{-\pi}^{-s \pi}+\int_{-s \pi}^{-r \pi}+\int_{-r \pi}^{-(1-r) \pi}+\int_{-(1-r) \pi}^{0}\right) G_{1,1}(\xi) d \xi \\
= & \int_{-\pi}^{-s \pi}\left(\frac{4}{3}-\frac{1}{3} h(\xi)\right) d \xi+\int_{-s \pi}^{-r \pi}\left(\frac{4}{3} h(\xi)-\frac{1}{3}\right) d \xi \\
& +\int_{-r \pi}^{-(1-r) \pi} d \xi+\int_{r \pi}^{\pi}\left(\frac{4}{3}-\frac{1}{3} h(\xi)\right) d \xi .
\end{aligned}
$$

Take

$$
h(\xi)= \begin{cases}4 & \text { on }[-\pi,-s \pi) \cup(r \pi, \pi] \\ 1 / 4 & \text { on }[-s \pi,-r \pi) \\ 1 & \text { on }[-r \pi, r \pi) .\end{cases}
$$

Then $S_{1,1}=\frac{1}{\pi} \int_{-\pi}^{0} G_{1,1}(\xi) d \xi=2 r-1<1$ so that the missing sample $c_{1}(f)(2 m)$ can be recovered. On the other hand, if we take $h(\xi)=\chi_{B}(\xi)$, then

$$
\begin{aligned}
\int_{-\pi}^{0} G_{1,1}(\xi) d \xi & =\int_{-\pi}^{-s \pi} \frac{4}{3} d \xi-\int_{-s \pi}^{-r \pi} \frac{1}{3} d \xi+\int_{-r \pi}^{-(1-r) \pi} d \xi+\int_{r \pi}^{\pi} \frac{4}{3} d \xi \\
& =\left(\frac{5}{3}+r-\frac{5}{3} s\right) \pi
\end{aligned}
$$

Hence, if $s=\frac{3}{5} r+\frac{2}{5}$, then $r<s<1$ and $S_{1,1}=\frac{1}{\pi} \int_{-\pi}^{0} G_{1,1}(\xi) d \xi=1$ so that the missing sample $c_{1}(f)(2 m)$ cannot be recovered by choosing $h(\xi)=\chi_{B}(\xi)$.

\section{Missing samples from both channels}

We assume $X_{1} \neq \phi$ and $X_{2} \neq \phi$ so that we are missing samples from both $c_{1}(f)(t)$ and $c_{2}(f)(t)$. In Section 4 we take $h(\xi)=\chi_{B}(\xi)$. Then

$$
\begin{aligned}
R_{j, k}(m, n) & =\left\langle\phi_{k, n}(\xi) \chi_{B}(\xi), \phi_{j, m}^{*}(\xi)\right\rangle_{L^{2}[-\pi, \pi]} \\
& =\left\langle\phi_{k, n}(\xi), \phi_{j, m}^{*}(\xi)\right\rangle_{L^{2}(B)} .
\end{aligned}
$$

Hence, we have for any $w=(u, v) \in \mathbb{C}^{M+N}$

$$
\begin{gathered}
\langle S w, w\rangle=\sum_{j=1}^{M} \sum_{k=1}^{M} R_{1,1}\left(m_{j}, m_{k}\right) u_{k} \overline{u_{j}}+\sum_{j=1}^{M} \sum_{k=1}^{N} R_{1,2}\left(m_{j}, n_{k}\right) v_{k} \overline{u_{j}} \\
+\sum_{j=1}^{N} \sum_{k=1}^{M} R_{2,1}\left(n_{j}, m_{k}\right) u_{k} \overline{v_{j}}+\sum_{j=1}^{N} \sum_{k=1}^{N} R_{2,2}\left(n_{j}, n_{k}\right) v_{k} \overline{v_{j}}
\end{gathered}
$$




$$
=\left\langle\sum_{k=1}^{M} u_{k} \phi_{1, m_{k}}(\xi)+\sum_{k=1}^{N} v_{k} \phi_{2, n_{k}}(\xi), \sum_{k=1}^{M} u_{k} \phi_{1, m_{k}}^{*}(\xi)+\sum_{k=1}^{N} v_{k} \phi_{2, n_{k}}^{*}(\xi)\right\rangle_{L^{2}(B)} .
$$

Since $\left(T^{*}\right)^{-1}\left(e_{j, n}(\xi)\right)=\phi_{j, n}(\xi)$ and so $T\left(e_{j, n}(\xi)\right)=\phi_{j, n}^{*}(\xi)(\mathrm{cf.}(2.5))$,

$$
\begin{aligned}
\langle S w, w\rangle=\left\langle\left(T^{-1}\right)^{*}(w(\xi)), T(w(\xi))\right\rangle_{L^{2}(B)} & =\left\langle P\left(T^{-1}\right)^{*}(w(\xi)), T(w(\xi))\right\rangle_{L^{2}[-\pi, \pi]} \\
& =\left\langle(w(\xi)), T^{-1} P T(w(\xi))\right\rangle_{L^{2}[-\pi, \pi]}
\end{aligned}
$$

where $P: L^{2}[-\pi, \pi] \longrightarrow L^{2}(B)$ is the orthogonal projection given by $P(\phi)(\xi)=$ $\phi(\xi) \chi_{B}(\xi)$ for any $\phi(\xi) \in L^{2}[-\pi, \pi]$ and

$$
w(\xi)=u(\xi)+v(\xi)=\sum_{k=1}^{M} u_{k} e_{1, m_{k}}(\xi)+\sum_{k=1}^{N} v_{k} e_{2, n_{k}}(\xi)
$$

Assume $P T=T P$ on $L^{2}[-\pi, \pi]$. Then

$$
\langle S w, w\rangle=\langle w(\xi), P(w(\xi))\rangle_{L^{2}[-\pi, \pi]}=\int_{B_{-}}|w(\xi)|^{2} d \xi+\int_{B_{+}}|w(\xi)|^{2} d \xi
$$

since $\operatorname{supp} u(\xi) \subseteq[-\pi, 0]$ and $\operatorname{supp} v(\xi) \subseteq[0, \pi]$, where

$$
B_{-}=B \cap[-\pi, 0], B_{+}=B \cap[0, \pi] .
$$

Hence, $\langle S w, w\rangle \leq \int_{-\pi}^{0}|w(\xi)|^{2} d \xi+\int_{0}^{\pi}|w(\xi)|^{2} d \xi=\|w\|^{2}$ for any $w$ in $\mathbb{C}^{M+N}$.

Lemma 4.1. Assume $P T=T P$ on $L^{2}[-\pi, \pi]$. Then $\langle S w, w\rangle<\|w\|^{2}$ for any $w \in \mathbb{C}^{M+N} \backslash\{0\}$ if and only if $\left|B_{-}\right|<\pi$ and $\left|B_{+}\right|<\pi$.

Proof. Assume that $\left|B_{ \pm}\right|<\pi$ and take $w=(u, v) \neq 0$. We may assume $u \neq 0$. Then $\int_{B_{-}}|u(\xi)|^{2} d \xi<\int_{-\pi}^{0}|u(\xi)|^{2} d \xi=\|u\|^{2}$ so that $\langle S w, w\rangle<\|w\|^{2}$.

Conversely, assume $\left|B_{-}\right|=\pi$ (then $\left|B_{+}\right|<\pi$ since $|B|<2 \pi$ ). Then for any $w=(u, 0)$ with $u \neq 0,\langle S w, w\rangle=\|u\|^{2}=\|w\|^{2}$.

The idea of using Lemma 4.1 in proving Theorem 4.2 follows from a result in [5] in which oversampling is handled in the abstract setting on general reproducing kernel Hilbert spaces.

Theorem 4.2. Let $B=B_{-} \cup B_{+}$be a band region with $B \subset[-\pi, \pi]$ and $\left|B_{ \pm}\right|<\pi$. Then for any signal $f(t)$ in $P W_{B}$, any finitely many mixed samples $\left\{c_{1}(f)\left(2 m_{k}\right): 1 \leq k \leq M\right\} \cup\left\{c_{2}(f)\left(2 n_{k}\right): 1 \leq k \leq N\right\}$ can be uniquely recovered if any one of the following 3 conditions holds:

(i) $B_{-}=B_{+}-\pi$;

(ii) $a_{1}(\xi)=0$ on $B_{+} \Delta\left(B_{-}+\pi\right)$ and $a_{2}(\xi)=0$ on $B_{-} \Delta\left(B_{+}-\pi\right)$;

(iii) $a_{1}(\xi)=0$ on $B_{-} \Delta\left(B_{+}-\pi\right)$ and $a_{2}(\xi)=0$ on $B_{+} \Delta\left(B_{-}+\pi\right)$.

Here $A \Delta B=(A \backslash B) \cup(B \backslash A)$ is the symmetric difference of $A$ and $B$. 
Proof. By lemma 4.1 any finitely many missing samples $\mathbf{f}$ can be uniquely recovered if $P T=T P$ and $\left|B_{ \pm}\right|<\pi$. On the other hand, we have for any $\phi$ in $L^{2}[-\pi, \pi]$

$$
P T(\phi)(\xi)= \begin{cases}\frac{1}{\sqrt{2}}\left[\overline{a_{1}(\xi)} \phi(\xi) \chi_{B_{-}}(\xi)+\overline{a_{2}(\xi)} \phi(\xi+\pi) \chi_{B_{-}}(\xi)\right] \quad, \quad-\pi \leq \xi<0 \\ \frac{1}{\sqrt{2}}\left[\overline{a_{1}(\xi)} \phi(\xi-\pi) \chi_{B_{+}}(\xi)+\overline{a_{2}(\xi)} \phi(\xi) \chi_{B_{+}}(\xi)\right] \quad, \quad 0 \leq \xi \leq \pi\end{cases}
$$

and

$T P(\phi)(\xi)= \begin{cases}\frac{1}{\sqrt{2}}\left[\overline{a_{1}(\xi)} \phi(\xi) \chi_{B_{-}}(\xi)+\overline{a_{2}(\xi)} \phi(\xi+\pi) \chi_{B_{+}-\pi}(\xi)\right], \quad-\pi \leq \xi<0 \\ \frac{1}{\sqrt{2}}\left[\overline{a_{1}(\xi)} \phi(\xi-\pi) \chi_{B_{-}+\pi}(\xi)+\overline{a_{2}(\xi)} \phi(\xi) \chi_{B_{+}}(\xi)\right], \quad 0 \leq \xi \leq \pi .\end{cases}$

Hence, $T P=P T$ on $L^{2}[-\pi, \pi]$ if and only if

$$
\left\{\begin{array}{l}
a_{2}(\xi)\left[\chi_{B_{-}}(\xi)-\chi_{B_{+}-\pi}(\xi)\right]=0 \text { on }[-\pi, 0] \\
a_{1}(\xi)\left[\chi_{B_{+}}(\xi)-\chi_{B_{-}+\pi}(\xi)\right]=0 \text { on }[0, \pi]
\end{array}\right.
$$

Now the condition (4.1) holds if and only if either (i) or (ii) in Theorem 4.2 holds. Therefore, under the condition (i) or (ii), $T P=P T$ on $L^{2}[-\pi, \pi]$ so that any mixed missing samples $\mathbf{f}$ can be uniquely recovered. Finally, exchanging the roles of $a_{1}(\xi)$ and $a_{2}(\xi)$ gives the condition (iii) under which $\mathbf{f}$ can also be uniquely recovered.

As a special case, if $B=[-r \pi,-(1-r) \pi] \cup[(1-r) \pi, r \pi]$ with $\frac{1}{2}<r<1$ is a band-pass region, then for any band-pass signal $f(t)$ in $P W_{B}$, any finitely many missing samples $\left\{c_{1}(f)\left(2 m_{k}\right): 1 \leq k \leq M\right\} \cup\left\{c_{2}(f)\left(2 n_{k}\right): 1 \leq k \leq N\right\}$ can be uniquely recovered.

Remark 4.1. Theorem 4.2 provides only sufficient conditions under which mixed missing samples can be recovered. For example, let us consider Example 3.1 again, in which none of the three conditions in Theorem 4.2 holds. We now assume that two mixed samples $f(2 p)$ and $f^{\prime}(2 p)$ are lost for some integer $p$. Then using $G_{1,1}(\xi), G_{2,2}(\xi)$ as in Example 3.1 and

$$
\begin{aligned}
& G_{1,2}(\xi)= \begin{cases}\frac{-i}{\pi} \quad,-\pi \leq \xi<-r \pi \\
0 \quad,-r \pi \leq \xi<-(1-r) \pi \\
\frac{i}{\pi},-(1-r) \pi \leq \xi \leq 0\end{cases} \\
& G_{2,1}(\xi)= \begin{cases}-i \xi\left(1+\frac{\xi}{\pi}\right) \quad,-\pi \leq \xi<-r \pi \\
0 \quad,-r \pi \leq \xi<-(1-r) \pi \\
i \xi\left(1+\frac{\xi}{\pi}\right) \quad,-(1-r) \pi \leq \xi \leq 0\end{cases}
\end{aligned}
$$


We have for any $w=(u, v)^{T} \neq 0$ in $\mathbb{C}^{2}$,

$$
\begin{aligned}
\langle S w, w\rangle & =\int_{-\pi}^{0}\left[G_{1,1}(\xi)|u|^{2}+G_{1,2}(\xi) u \bar{v}+G_{2,1}(\xi) \bar{u} v+G_{2,2}(\xi)|v|^{2}\right] d \xi \\
& =\int_{-\pi}^{0}\left[G_{1,1}(\xi)|u|^{2}+G_{2,2}(\xi)|v|^{2}\right] d \xi \\
& <|u|^{2}+|v|^{2}=\|w\|^{2}
\end{aligned}
$$

since $\int_{-\pi}^{0} G_{1,2}(\xi)=\int_{-\pi}^{0} G_{2,1}(\xi)=0$. Therefore, missing samples $f(2 p)$ and $f^{\prime}(2 p)$ can be recovered.

Likewise, in the case of Hilbert transform sampling as in Example 3.2, one can show that if $p$ and $q$ are integers such that $2(p-q) r$ is not an odd integer, then missing samples $f(2 p)$ and $f^{\prime}(2 p)$ can be recovered although none of the three conditions in Theorem 4.2 holds.

\section{ACKNOWLEDGEMENT}

This work is partially supported by KOSEF(R01-2006-000-10424-0). Authors are grateful to the referees for their many valuable comments, which improve the paper.

\section{References}

[1] P. J. S. G. Ferreira, Incomplete sampling series and the recovery of missing samples from oversampled band-limited signals, IEEE Trans. Signal Processing, 40, 225-227, 1992.

[2] P. J. S. G. Ferreira, The stability of a procedure for the recovery of lost samples in band-limited signals, Signal Proc., 40, 195-205, 1994.

[3] J. R. Higgins, Sampling theory for Paley-Wiener spaces in the Riesz basis setting, Proc. R. Irish Acad., 94A, 219-236, 1994.

[4] J. R. Higgins, Sampling Theory in Fourier and Signal Analysis, Oxford University Press, Oxford, 1996.

[5] Y. M. Hong, J. M. Kim and K. H. Kwon, Sampling theory in abstract reproducing kernel Hilbert space, Sampl. Theory Signal Image Process., 6, 109-121, 2007.

[6] J. M. Kim and K. H. Kwon, Vector sampling expansion in Riesz basis setting and its aliasing error, preprint. 
[7] R. J. Marks II, Introduction to Shannon Sampling and Interpolation Theory, Springer-Verlag, New-York, 1991.

[8] A. Papoulis, Generalized sampling expansion, IEEE Trans. on Circuits and Systems, 24, 652-654, 1977.

[9] D. M. S. Santos and P. J. S. G. Ferreira, Reconstruction from missing function and derivative samples and oversampled filter banks, Proceedings of the IEEE International Conference on Acoustics, Speech, and Signal Processing, ICASSP 2004, 3, 941-944, 2004. 